\title{
Hybrid Proximal-Type Algorithms for Generalized Equilibrium Problems, Maximal Monotone Operators, and Relatively Nonexpansive Mappings
}

\author{
Lu-Chuan Zeng, ${ }^{1,2}$ Q. H. Ansari, ${ }^{3}$ and S. Al-Homidan ${ }^{3}$ \\ ${ }^{1}$ Department of Mathematics, Shanghai Normal University, Shanghai 200234, China \\ ${ }^{2}$ Scientific Computing Key Laboratory of Shanghai Universities, Shanghai 200234, China \\ ${ }^{3}$ Department of Mathematics and Statistics, King Fahd University of Petroleum \& Minerals (KFUPM), \\ P.O. Box 119, Dhahran 31261, Saudi Arabia
}

Correspondence should be addressed to S. Al-Homidan, homidan@kfupm.edu.sa

Received 24 September 2010; Accepted 18 October 2010

Academic Editor: Jen Chih Yao

Copyright (c) $2011 \mathrm{Lu}$-Chuan Zeng et al. This is an open access article distributed under the Creative Commons Attribution License, which permits unrestricted use, distribution, and reproduction in any medium, provided the original work is properly cited.

\begin{abstract}
The purpose of this paper is to introduce and consider new hybrid proximal-type algorithms for finding a common element of the set EP of solutions of a generalized equilibrium problem, the set $F(S)$ of fixed points of a relatively nonexpansive mapping $S$, and the set $T^{-1} 0$ of zeros of a maximal monotone operator $T$ in a uniformly smooth and uniformly convex Banach space. Strong convergence theorems for these hybrid proximal-type algorithms are established; that is, under appropriate conditions, the sequences generated by these various algorithms converge strongly to the same point in $\mathrm{EP} \cap F(S) \cap T^{-1} 0$. These new results represent the improvement, generalization, and development of the previously known ones in the literature.
\end{abstract}

\section{Introduction}

Let $E$ be a real Banach space with the dual $E^{*}$ and $C$ be a nonempty closed convex subset of $E$. We denote by $\mathcal{N}$ and $\mathcal{R}$ the sets of positive integers and real numbers, respectively. Also, we denote by $J$ the normalized duality mapping from $E$ to $2^{E^{*}}$ defined by

$$
J x=\left\{x^{*} \in E^{*}:\left\langle x, x^{*}\right\rangle=\|x\|^{2}=\left\|x^{*}\right\|^{2}\right\}, \quad \forall x \in E,
$$

where $\langle\cdot, \cdot\rangle$ denotes the generalized duality pairing. Recall that if $E$ is smooth, then $J$ is single valued and if $E$ is uniformly smooth, then $J$ is uniformly norm-to-norm continuous on bounded subsets of $E$. We will still denote by $J$ the single valued duality mapping. Let 
$f: C \times C \rightarrow R$ be a bifunction and $A: C \rightarrow E^{*}$ be a nonlinear mapping. We consider the following generalized equilibrium problem:

$$
\text { find } u \in C \text { such that } f(u, y)+\langle A u, y-u\rangle \geq 0, \quad \forall y \in C \text {. }
$$

The set of such $u \in C$ is denoted by EP, that is,

$$
\mathrm{EP}=\{u \in C: f(u, y)+\langle A u, y-u\rangle \geq 0, \forall y \in C\}
$$

Whenever $E=H$ a Hilbert space, problem (1.2) was introduced and studied by S. Takahashi and W. Takahashi [1]. Similar problems have been studied extensively recently. See, for example, [2-11]. In the case of $A \equiv 0, \mathrm{EP}$ is denoted by $\mathrm{EP}(f)$. In the case of $f \equiv 0, \mathrm{EP}$ is also denoted by $\operatorname{VI}(C, A)$. The problem (1.2) is very general in the sense that it includes, as special cases, optimization problems, variational inequalities, minimax problems, the Nash equilibrium problem in noncooperative games and others; see, for example, [12-14]. A mapping $S: C \rightarrow E$ is called nonexpansive if $\|S x-S y\| \leq\|x-y\|$ for all $x, y \in C$. Denote by $F(S)$ the set of fixed points of $S$, that is, $F(S)=\{x \in C: S x=x\}$. A mapping $A: C \rightarrow E^{*}$ is called $\alpha$-inverse-strongly monotone, if there exists an $\alpha>0$ such that

$$
\langle A x-A y, x-y\rangle \geq \alpha\|A x-A y\|^{2}, \quad \forall x, y \in C .
$$

It is easy to see that if $A: C \rightarrow E^{*}$ is an $\alpha$-inverse-strongly monotone mapping, then it is $1 / \alpha$-Lipschitzian.

Let $E$ be a real Banach space with the dual $E^{*}$. A multivalued operator $T: E \rightarrow 2^{E^{*}}$ with domain $D(T)=\{z \in E: T z \neq \emptyset\}$ is called monotone if $\left\langle x_{1}-x_{2}, y_{1}-y_{2}\right\rangle \geq 0$ for each $x_{i} \in D(T)$ and $y_{i} \in T x_{i}, i=1,2$. A monotone operator $T$ is called maximal if its graph $G(T)=\{(x, y): y \in T x\}$ is not properly contained in the graph of any other monotone operator. A method for solving the inclusion $0 \in T x$ is the proximal point algorithm. Denote by $I$ the identity operator on $E=H$ a Hilbert space. The proximal point algorithm generates, for any initial point $x_{0}=x \in H$, a sequence $\left\{x_{n}\right\}$ in $H$, by the iterative scheme

$$
x_{n+1}=\left(I+r_{n} T\right)^{-1} x_{n}, \quad n=0,1,2, \ldots,
$$

where $\left\{r_{n}\right\}$ is a sequence in the interval $(0, \infty)$. Note that this iteration is equivalent to

$$
0 \in T x_{n+1}+\frac{1}{r_{n}}\left(x_{n+1}-x_{n}\right), \quad n=0,1,2, \ldots
$$

This algorithm was first introduced by Martinet [12] and generally studied by Rockafellar [15] in the framework of a Hilbert space. Later many authors studied its convergence in a Hilbert space or a Banach space. See, for instance, [16-21] and the references therein.

Let $E$ be a reflexive, strictly convex, and smooth Banach space with the dual $E^{*}$ and $C$ be a nonempty closed convex subset of $E$. Let $T: E \rightarrow 2^{E^{*}}$ be a maximal monotone operator with domain $D(T)=C$ and $S: C \rightarrow C$ be a relatively nonexpansive mapping. Let $A: C \rightarrow$ $X^{*}$ be an $\alpha$-inverse-strongly monotone mapping and $f: C \times C \rightarrow R$ be a bifunction satisfying 
(A1)-(A4): (A1) $f(x, x)=0, \forall x \in C$; (A2) $f$ is monotone, that is, $f(x, y)+f(y, x) \leq 0$, $\forall x, y \in C$; (A3) $\limsup _{t \downarrow 0} f(x+t(z-x), y) \leq f(x, y), \forall x, y, z \in C$; (A4) the function $y \mapsto$ $f(x, y)$ is convex and lower semicontinuous. The purpose of this paper is to introduce and investigate two new hybrid proximal-type Algorithms 1.1 and 1.2 for finding an element of $E P \cap F(S) \cap T^{-1} 0$.

Algorithm 1.1.

$$
\begin{gathered}
x_{0} \in C \text { arbitrarily chosen, } \\
0=v_{n}+\frac{1}{r_{n}}\left(J \tilde{x}_{n}-J x_{n}\right), \quad v_{n} \in T \tilde{x}_{n}, \\
z_{n}=J^{-1}\left(\beta_{n} J \tilde{x}_{n}+\left(1-\beta_{n}\right) J S \tilde{x}_{n}\right), \\
y_{n}=J^{-1}\left(\alpha_{n} J \tilde{x}_{n}+\left(1-\alpha_{n}\right) J S z_{n}\right), \\
u_{n} \in C \text { such that } \\
f\left(u_{n}, y\right)+\left\langle A u_{n}, y-u_{n}\right\rangle+\frac{1}{r_{n}}\left\langle y-u_{n}, J u_{n}-J y_{n}\right\rangle \geq 0, \quad \forall y \in C, \\
H_{n}=\left\{v \in C: \phi\left(v, u_{n}\right) \leq \alpha_{n} \phi\left(v, \tilde{x}_{n}\right)+\left(1-\alpha_{n}\right) \phi\left(v, z_{n}\right),\left\langle v-\tilde{x}_{n}, v_{n}\right\rangle \leq 0\right\}, \\
W_{n}=\left\{v \in C:\left\langle v-x_{n}, J x_{0}-J x_{n}\right\rangle \leq 0\right\}, \\
x_{n+1}=\Pi_{H_{n} \cap W_{n}} x_{0}, \quad n=0,1,2, \ldots,
\end{gathered}
$$

where $\left\{r_{n}\right\}_{n=0}^{\infty}$ is a sequence in $(0, \infty)$ and $\left\{\alpha_{n}\right\}_{n=0}^{\infty},\left\{\beta_{n}\right\}_{n=0}^{\infty}$ are sequences in $[0,1]$.

Algorithm 1.2.

$$
\begin{gathered}
x_{0} \in C \text { arbitrarily chosen, } \\
0=v_{n}+\frac{1}{r_{n}}\left(J \tilde{x}_{n}-J x_{n}\right), \quad v_{n} \in T \tilde{x}_{n}, \\
y_{n}=J^{-1}\left(\alpha_{n} J x_{0}+\left(1-\alpha_{n}\right) J S \tilde{x}_{n}\right), \\
u_{n} \in C \text { such that } \\
f\left(u_{n}, y\right)+\left\langle A u_{n}, y-u_{n}\right\rangle+\frac{1}{r_{n}}\left\langle y-u_{n}, J u_{n}-J y_{n}\right\rangle \geq 0, \quad \forall y \in C, \\
H_{n}=\left\{v \in C: \phi\left(v, u_{n}\right) \leq \alpha_{n} \phi\left(v, x_{0}\right)+\left(1-\alpha_{n}\right) \phi\left(v, \tilde{x}_{n}\right),\left\langle v-\tilde{x}_{n}, v_{n}\right\rangle \leq 0\right\}, \\
W_{n}=\left\{v \in C:\left\langle v-x_{n}, J x_{0}-J x_{n}\right\rangle \leq 0\right\}, \\
x_{n+1}=\prod_{H_{n} \cap W_{n}} x_{0}, \quad n=0,1,2, \ldots,
\end{gathered}
$$

where $\left\{r_{n}\right\}_{n=0}^{\infty}$ is a sequence in $(0, \infty)$ and $\left\{\alpha_{n}\right\}_{n=0}^{\infty}$ is a sequence in $[0,1]$. 
In this paper, strong convergence results on these two hybrid proximal-type algorithms are established; that is, under appropriate conditions, the sequence $\left\{x_{n}\right\}$ generated by Algorithm 1.1 and the sequence $\left\{x_{n}\right\}$ generated by Algorithm 1.2, converge strongly to the same point $\Pi_{\operatorname{EP} \cap F(S) \cap T^{-1} 0} x_{0}$. These new results represent the improvement, generalization and development of the previously known ones in the literature including Solodov and Svaiter [22], Kamimura and Takahashi [23], Qin and Su [24], and Ceng et al. [25].

Throughout this paper the symbol $\rightarrow$ stands for weak convergence and $\rightarrow$ stands for strong convergence.

\section{Preliminaries}

Let $E$ be a real Banach space with the dual $E^{*}$. We denote by $J$ the normalized duality mapping from $E$ to $2^{E^{*}}$ defined by

$$
J x=\left\{x^{*} \in E^{*}:\left\langle x, x^{*}\right\rangle=\|x\|^{2}=\left\|x^{*}\right\|^{2}\right\}, \quad \forall x \in X,
$$

where $\langle\cdot, \cdot\rangle$ denotes the generalized duality pairing. A Banach space $E$ is called strictly convex if $\|(x+y) / 2\|<1$ for all $x, y \in E$ with $\|x\|=\|y\|=1$ and $x \neq y$. It is said to be uniformly convex if $x_{n}-y_{n} \rightarrow 0$ for any two sequences $\left\{x_{n}\right\},\left\{y_{n}\right\} \subset E$ such that $\left\|x_{n}\right\|=\left\|y_{n}\right\|=1$ and $\lim _{n \rightarrow \infty}\left\|\left(x_{n}+y_{n}\right) / 2\right\|=1$. Let $U=\{x \in E:\|x\|=1\}$ be a unit sphere of $E$, then the Banach space $E$ is called smooth if

$$
\lim _{t \rightarrow 0} \frac{\|x+t y\|-\|x\|}{t}
$$

exists for each $x, y \in U$. If $E$ is smooth, then $J$ is single valued. We still denote the single valued duality mapping by $J$.

It is also said to be uniformly smooth if the limit is attained uniformly for $x, y \in U$. Recall also that if $E$ is uniformly smooth, then $J$ is uniformly norm-to-norm continuous on bounded subsets of $E$. A Banach space $E$ is said to have the Kadec-Klee property if for any sequence $\left\{x_{n}\right\} \subset E$, whenever $x_{n} \rightarrow x \in E$ and $\left\|x_{n}\right\| \rightarrow\|x\|$, we have $x_{n} \rightarrow x$. It is known that if $E$ is uniformly convex, then $E$ has the Kadec-Klee property; see $[26,27]$ for more details.

Let $C$ be a nonempty closed convex subset of a real Hilbert space $H$ and $P_{C}: H \rightarrow C$ be the metric projection of $H$ onto $C$, then $P_{C}$ is nonexpansive. This fact actually characterizes Hilbert spaces and hence, it is not available in more general Banach spaces. Nevertheless, Alber [28] recently introduced a generalized projection operator $\Pi_{C}$ in a Banach space $E$ which is an analogue of the metric projection in Hilbert spaces.

Next, we assume that $E$ is a smooth Banach space. Consider the functional defined as in $[28,29]$ by

$$
\phi(x, y)=\|x\|^{2}-2\langle x, J y\rangle+\|y\|^{2}, \quad \forall x, y \in E
$$

It is clear that in a Hilbert space $H,(2.3)$ reduces to $\phi(x, y)=\|x-y\|^{2}$, for all $x, y \in H$. 
The generalized projection $\Pi_{C}: E \rightarrow C$ is a mapping that assigns to an arbitrary point $x \in E$ the minimum point of the functional $\phi(y, x)$; that is, $\Pi_{C} x=\bar{x}$, where $\bar{x}$ is the solution to the minimization problem

$$
\phi(\bar{x}, x)=\min _{y \in C} \phi(y, x) .
$$

The existence and uniqueness of the operator $\Pi_{C}$ follows from the properties of the functional $\phi(x, y)$ and strict monotonicity of the mapping $J$ (see, e.g., [30]). In a Hilbert space $H, \Pi_{C}=$ $P_{C}$. From [28], in uniformly smooth and uniformly convex Banach spaces, we have

$$
(\|x\|-\|y\|)^{2} \leq \phi(x, y) \leq(\|x\|+\|y\|)^{2}, \quad \forall x, y \in E .
$$

Let $C$ be a nonempty closed convex subset of $E$, and let $S$ be a mapping from $C$ into itself. A point $p \in C$ is called an asymptotically fixed point of $S$ [31] if $C$ contains a sequence $\left\{x_{n}\right\}$ which converges weakly to $p$ such that $S x_{n}-x_{n} \rightarrow 0$. The set of asymptotical fixed points of $S$ will be denoted by $\widehat{F}(S)$. A mapping $S$ from $C$ into itself is called relatively nonexpansive [32-34] if $\widehat{F}(S)=F(S)$ and $\phi(p, S x) \leq \phi(p, x)$ for all $x \in C$ and $p \in F(S)$.

We remark that if $E$ is a reflexive, strictly convex and smooth Banach space, then for any $x, y \in E, \phi(x, y)=0$ if and only if $x=y$. It is sufficient to show that if $\phi(x, y)=0$ then $x=y$. From (2.5), we have $\|x\|=\|y\|$. This implies that $\langle x, J y\rangle=\|x\|^{2}=\|y\|^{2}$. From the definition of $J$, we have $J x=J y$. Therefore, we have $x=y$; see [26, 27] for more details.

We need the following lemmas for the proof of our main results.

Lemma 2.1 (see [23]). Let $E$ be a smooth and uniformly convex Banach space and let $\left\{x_{n}\right\}$ and $\left\{y_{n}\right\}$ be two sequences of $E$. If $\phi\left(x_{n}, y_{n}\right) \rightarrow 0$ and either $\left\{x_{n}\right\}$ or $\left\{y_{n}\right\}$ is bounded, then $x_{n}-y_{n} \rightarrow 0$.

Lemma 2.2 (see $[23,28]$ ). Let $C$ be a nonempty closed convex subset of a smooth, strictly convex and reflexive Banach space $E$, let $x \in E$ and let $z \in C$, then

$$
z=\Pi_{C} x \Longleftrightarrow\langle y-z, J x-J z\rangle \leq 0, \quad \forall y \in C
$$

Lemma 2.3 (see $[23,28]$ ). Let $C$ be a nonempty closed convex subset of a smooth, strictly convex and reflexive Banach space $E$, then

$$
\phi\left(x, \Pi_{C} y\right)+\phi\left(\Pi_{C} y, y\right) \leq \phi(x, y), \quad \forall x \in C, y \in E
$$

Lemma 2.4 (see [35]). Let $C$ be a nonempty closed convex subset of a reflexive, strictly convex and smooth Banach space $E$, and let $S: C \rightarrow C$ be a relatively nonexpansive mapping, then $F(S)$ is closed and convex.

The following result is according to Blum and Oettli [36]. 
Lemma 2.5 (see [36]). Let $C$ be a nonempty closed convex subset of a smooth, strictly convex and reflexive Banach space $E$, let $f$ be a bifunction from $C \times C$ to $R$ satisfying (A1)-(A4), and let $r>0$ and $x \in E$, then, there exists $z \in C$ such that

$$
f(z, y)+\frac{1}{r}\langle y-z, J z-J x\rangle \geq 0, \quad \forall y \in C
$$

Motivated by Combettes and Hirstoaga [37] in a Hilbert space, Takahashi and Zembayashi [38] established the following lemma.

Lemma 2.6 (see [38]). Let $C$ be a nonempty closed convex subset of a uniformly smooth, strictly convex and reflexive Banach space $E$, and let $f$ be a bifunction from $C \times C$ to $R$ satisfying (A1)-(A4). For $r>0$ and $x \in E$, define a mapping $T_{r}: E \rightarrow C$ as follows:

$$
T_{r}(x)=\left\{z \in C: f(z, y)+\frac{1}{r}\langle y-z, J z-J x\rangle \geq 0, \forall y \in C\right\}
$$

for all $x \in E$, then, the following hold:

(i) $T_{r}$ is single valued;

(ii) $T_{r}$ is a firmly nonexpansive-type mapping, that is, for all $x, y \in E$,

$$
\left\langle T_{r} x-T_{r} y, J T_{r} x-J T_{r} y\right\rangle \leq\left\langle T_{r} x-T_{r} y, J x-J y\right\rangle
$$

(iii) $F\left(T_{r}\right)=\widehat{F}\left(T_{r}\right)=\mathrm{EP}(f)$;

(iv) $\mathrm{EP}(f)$ is closed and convex.

Using Lemma 2.6, one has the following result.

Lemma 2.7 (see [38]). Let $C$ be a nonempty closed convex subset of a smooth, strictly convex and reflexive Banach space $E$, let $f$ be a bifunction from $C \times C$ to $R$ satisfying $(A 1)-(A 4)$, and let $r>0$, then, for $x \in E$ and $q \in F\left(T_{r}\right)$,

$$
\phi\left(q, T_{r} x\right)+\phi\left(T_{r} x, x\right) \leq \phi(q, x)
$$

Utilizing Lemmas 2.5, 2.6 and 2.7 as above, Chang [39] derived the following result.

Proposition 2.8 (see [39, Lemma 2.5]). Let E be a smooth, strictly convex and reflexive Banach space and $C$ be a nonempty closed convex subset of E. Let $A: C \rightarrow E^{*}$ be an $\alpha$-inverse-strongly monotone mapping, let $f$ be a bifunction from $C \times C$ to $R$ satisfying (A1)-(A4), and let $r>0$, then there hold the following: 
Fixed Point Theory and Applications

(I) for $x \in E$, there exists $u \in C$ such that

$$
f(u, y)+\langle A u, y-u\rangle+\frac{1}{r}\langle y-u, J u-J x\rangle \geq 0, \quad \forall y \in C
$$

(II) if $E$ is additionally uniformly smooth and $K_{r}: E \rightarrow C$ is defined as

$$
K_{r}(x)=\left\{u \in C: f(u, y)+\langle A u, y-u\rangle+\frac{1}{r}\langle y-u, J u-J x\rangle \geq 0, \forall y \in C\right\}, \quad \forall x \in E,
$$

then the mapping $K_{r}$ has the following properties:

(i) $K_{r}$ is single valued,

(ii) $K_{r}$ is a firmly nonexpansive-type mapping, that is,

$$
\left\langle K_{r} x-K_{r} y, J K_{r} x-J K_{r} y\right\rangle \leq\left\langle K_{r} x-K_{r} y, J x-J y\right\rangle, \quad \forall x, y \in E,
$$

(iii) $F\left(K_{r}\right)=\widehat{F}\left(K_{r}\right)=\mathrm{EP}$,

(iv) $\mathrm{EP}$ is a closed convex subset of $C$,

(v) $\phi\left(p, K_{r} x\right)+\phi\left(K_{r} x, x\right) \leq \phi(p, x)$, for all $p \in F\left(K_{r}\right)$.

Proof. Define a bifunction $F: C \times C \rightarrow R$ as follows:

$$
F(x, y)=f(x, y)+\langle A x, y-x\rangle, \quad \forall x, y \in C .
$$

Then it is easy to verify that $F$ satisfies the conditions (A1)-(A4). Therefore, The conclusions (I) and (II) of Proposition 2.8 follow immediately from Lemmas 2.5, 2.6 and 2.7.

Lemma 2.9 (see $[13,14])$. Let $E$ be a reflexive, strictly convex and smooth Banach space, and let $T: E \rightarrow 2^{E^{*}}$ be a maximal monotone operator with $T^{-1} 0 \neq \emptyset$, then,

$$
\phi\left(z, J_{r} x\right)+\phi\left(J_{r} x, x\right) \leq \phi(z, x), \quad \forall r>0, z \in T^{-1} 0, x \in E .
$$

\section{Main Results}

Throughout this section, unless otherwise stated, we assume that $T: E \rightarrow 2^{E^{*}}$ is a maximal monotone operator with domain $D(T)=C, S: C \rightarrow C$ is a relatively nonexpansive mapping, $A: C \rightarrow E^{*}$ is an $\alpha$-inverse-strongly monotone mapping and $f: C \times C \rightarrow \mathcal{R}$ is a bifunction satisfying (A1)-(A4), where $C$ is a nonempty closed convex subset of a reflexive, strictly convex, and smooth Banach space $E$. In this section, we study the following algorithm. 
Algorithm 3.1.

$$
\begin{gathered}
x_{0} \in C \text { arbitrarily chosen, } \\
0=v_{n}+\frac{1}{r_{n}}\left(J \tilde{x}_{n}-J x_{n}\right), \quad v_{n} \in T \tilde{x}_{n}, \\
z_{n}=J^{-1}\left(\beta_{n} J \tilde{x}_{n}+\left(1-\beta_{n}\right) J S \tilde{x}_{n}\right), \\
y_{n}=J^{-1}\left(\alpha_{n} J \tilde{x}_{n}+\left(1-\alpha_{n}\right) J S z_{n}\right), \\
u_{n} \in C \text { such that } \\
f\left(u_{n}, y\right)+\left\langle A u_{n}, y-u_{n}\right\rangle+\frac{1}{r_{n}}\left\langle y-u_{n}, J u_{n}-J y_{n}\right\rangle \geq 0, \quad \forall y \in C, \\
H_{n}=\left\{v \in C: \phi\left(v, u_{n}\right) \leq \alpha_{n} \phi\left(v, \tilde{x}_{n}\right)+\left(1-\alpha_{n}\right) \phi\left(v, z_{n}\right),\left\langle v-\tilde{x}_{n}, v_{n}\right\rangle \leq 0\right\}, \\
W_{n}=\left\{v \in C:\left\langle v-x_{n}, J x_{0}-J x_{n}\right\rangle \leq 0\right\}, \\
x_{n+1}=\prod_{H_{n} \cap W_{n}} x_{0}, \quad n=0,1,2, \ldots,
\end{gathered}
$$

where $\left\{r_{n}\right\}_{n=0}^{\infty}$ is a sequence in $(0, \infty)$ and $\left\{\alpha_{n}\right\}_{n=0}^{\infty},\left\{\beta_{n}\right\}_{n=0}^{\infty}$ are sequences in $[0,1]$.

First we investigate the condition under which the Algorithm 3.1 is well defined. Rockafellar [40] proved the following result.

Lemma 3.2 (Rockafellar [40]). Let E be a reflexive, strictly convex, and smooth Banach space and let $T: E \rightarrow 2^{E^{*}}$ be a multivalued operator, then there hold the following:

(i) $T^{-1} 0$ is closed and convex if $T$ is maximal monotone such that $T^{-1} 0 \neq \emptyset$;

(ii) $T$ is maximal monotone if and only if $T$ is monotone with $R(J+r T)=E^{*}$ for all $r>0$.

Utilizing this result, we can show the following lemma.

Lemma 3.3. Let $E$ be a reflexive, strictly convex, and smooth Banach space. If $\mathrm{EP} \cap F(S) \cap T^{-1} 0 \neq \emptyset$, then the sequence $\left\{x_{n}\right\}$ generated by Algorithm 3.1 is well defined.

Proof. For each $n \geq 0$, define two sets $C_{n}$ and $D_{n}$ as follows:

$$
\begin{gathered}
C_{n}=\left\{v \in C: \phi\left(v, u_{n}\right) \leq \alpha_{n} \phi\left(v, \tilde{x}_{n}\right)+\left(1-\alpha_{n}\right) \phi\left(v, z_{n}\right)\right\}, \\
D_{n}=\left\{v \in C:\left\langle v-\tilde{x}_{n}, v_{n}\right\rangle \leq 0\right\} .
\end{gathered}
$$

It is obvious that $C_{n}$ is closed and $D_{n}, W_{n}$ are closed convex sets for each $n \geq 0$. Let us show that $C_{n}$ is convex. For $v_{1}, v_{2} \in C_{n}$ and $t \in(0,1)$, put $v=t v_{1}+(1-t) v_{2}$. It is sufficient to show that $v \in C_{n}$. Indeed, observe that

$$
\phi\left(v, u_{n}\right) \leq \alpha_{n} \phi\left(v, \tilde{x}_{n}\right)+\left(1-\alpha_{n}\right) \phi\left(v, z_{n}\right)
$$


is equivalent to

$$
2 \alpha_{n}\left\langle v, J \tilde{x}_{n}\right\rangle+2\left(1-\alpha_{n}\right)\left\langle v, J z_{n}\right\rangle-2\left\langle v, J u_{n}\right\rangle \leq \alpha_{n}\left\|\tilde{x}_{n}\right\|^{2}+\left(1-\alpha_{n}\right)\left\|z_{n}\right\|^{2}-\left\|u_{n}\right\|^{2} .
$$

Note that there hold the following:

$$
\begin{aligned}
& \phi\left(v, u_{n}\right)=\|v\|^{2}-2\left\langle v, J u_{n}\right\rangle+\left\|u_{n}\right\|^{2}, \\
& \phi\left(v, \tilde{x}_{n}\right)=\|v\|^{2}-2\left\langle v, J \tilde{x}_{n}\right\rangle+\left\|\tilde{x}_{n}\right\|^{2}, \\
& \phi\left(v, z_{n}\right)=\|v\|^{2}-2\left\langle v, J z_{n}\right\rangle+\left\|z_{n}\right\|^{2},
\end{aligned}
$$

Thus we have

$$
\begin{aligned}
& 2 \alpha_{n}\left\langle v, J \tilde{x}_{n}\right\rangle+2\left(1-\alpha_{n}\right)\left\langle v, J z_{n}\right\rangle-2\left\langle v, J u_{n}\right\rangle \\
&=2 \alpha_{n}\left\langle t v_{1}+(1-t) v_{2}, J \tilde{x}_{n}\right\rangle+2\left(1-\alpha_{n}\right)\left\langle t v_{1}+(1-t) v_{2}, J z_{n}\right\rangle \\
&-2\left\langle t v_{1}+(1-t) v_{2}, J u_{n}\right\rangle \\
&= 2 t \alpha_{n}\left\langle v_{1}, J \tilde{x}_{n}\right\rangle+2(1-t) \alpha_{n}\left\langle v_{2}, J \tilde{x}_{n}\right\rangle+2\left(1-\alpha_{n}\right) t\left\langle v_{1}, J z_{n}\right\rangle \\
&+2\left(1-\alpha_{n}\right)(1-t)\left\langle v_{2}, J z_{n}\right\rangle-2 t\left\langle v_{1}, J u_{n}\right\rangle-2(1-t)\left\langle v_{2}, J u_{n}\right\rangle \\
& \leq \alpha_{n}\left\|\tilde{x}_{n}\right\|^{2}+\left(1-\alpha_{n}\right)\left\|z_{n}\right\|^{2}-\left\|u_{n}\right\|^{2} .
\end{aligned}
$$

This implies that $v \in C_{n}$. Therefore, $C_{n}$ is convex and hence $H_{n}=C_{n} \cap D_{n}$ is closed and convex.

On the other hand, let $w \in \mathrm{EP} \cap F(S) \cap T^{-1} 0$ be arbitrarily chosen, then $w \in \mathrm{EP}, w \in$ $F(S)$ and $w \in T^{-1} 0$. From Algorithm 3.1, it follows that

$$
\begin{aligned}
\phi\left(w, u_{n}\right) & =\phi\left(w, K_{r_{n}} y_{n}\right) \leq \phi\left(w, y_{n}\right) \\
& =\phi\left(w, J^{-1}\left(\alpha_{n} J \tilde{x}_{n}+\left(1-\alpha_{n}\right) J S z_{n}\right)\right) \\
& =\|w\|^{2}-2\left\langle w, \alpha_{n} J \tilde{x}_{n}+\left(1-\alpha_{n}\right) J S z_{n}\right\rangle+\left\|\alpha_{n} J \tilde{x}_{n}+\left(1-\alpha_{n}\right) J S z_{n}\right\|^{2} \\
& \leq\|w\|^{2}-2 \alpha_{n}\left\langle w, J \tilde{x}_{n}\right\rangle-2\left(1-\alpha_{n}\right)\left\langle w, J S z_{n}\right\rangle+\alpha_{n}\left\|\tilde{x}_{n}\right\|^{2}+\left(1-\alpha_{n}\right)\left\|S z_{n}\right\|^{2} \\
& \leq \alpha_{n} \phi\left(w, \tilde{x}_{n}\right)+\left(1-\alpha_{n}\right) \phi\left(w, S z_{n}\right) \\
& \leq \alpha_{n} \phi\left(w, \tilde{x}_{n}\right)+\left(1-\alpha_{n}\right) \phi\left(w, z_{n}\right) .
\end{aligned}
$$

So $w \in C_{n}$ for all $n \geq 0$. Now, from Lemma 3.2 it follows that there exists $\left(\tilde{x}_{0}, v_{0}\right) \in E \times E^{*}$ such that $0=v_{0}+\left(1 / r_{0}\right)\left(J \tilde{x}_{0}-J x_{0}\right)$ and $v_{0} \in T \tilde{x}_{0}$. Since $T$ is monotone, it follows that $\left\langle\tilde{x}_{0}-w, v_{0}\right\rangle \geq 0$, which implies that $w \in D_{0}$ and hence $w \in H_{0}$. Furthermore, it is clear that $w \in W_{0}=C$, then $w \in H_{0} \cap W_{0}$, and therefore $x_{1}=\Pi_{H_{0} \cap W_{0}} x_{0}$ is well defined. Suppose that $w \in H_{n-1} \cap W_{n-1}$ and $x_{n}$ is well defined for some $n \geq 1$. Again by Lemma 3.2, we deduce that $\left(\tilde{x}_{n}, v_{n}\right) \in E \times E^{*}$ such that $0=v_{n}+\left(1 / r_{n}\right)\left(J \tilde{x}_{n}-J x_{n}\right)$ and $v_{n} \in T \tilde{x}_{n}$, then from the monotonicity of $T$ we 
conclude that $\left\langle\tilde{x}_{n}-w, v_{n}\right\rangle \geq 0$, which implies that $w \in D_{n}$ and hence $w \in H_{n}$. It follows from Lemma 2.4 that

$$
\left\langle w-x_{n}, J x_{0}-J x_{n}\right\rangle=\left\langle w-\Pi_{H_{n-1} \cap W_{n-1}} x_{0}, J x_{0}-J \Pi_{H_{n-1} \cap W_{n-1}} x_{0}\right\rangle \leq 0,
$$

which implies that $w \in W_{n}$. Consequently, $w \in H_{n} \cap W_{n}$ and so $\operatorname{EP} \cap F(S) \cap T^{-1} 0 \subset H_{n} \cap W_{n}$. Therefore $x_{n+1}=\Pi_{H_{n} \cap W_{n}} x_{0}$ is well defined, then, by induction, the sequence $\left\{x_{n}\right\}$ generated by Algorithm 3.1, is well defined for each integer $n \geq 0$.

Remark 3.4. From the above proof, we obtain that

$$
\mathrm{EP} \cap F(S) \cap T^{-1} 0 \subset H_{n} \cap W_{n}
$$

for each integer $n \geq 0$.

We are now in a position to prove the main theorem.

Theorem 3.5. Let E be a uniformly smooth and uniformly convex Banach space. Let $\left\{r_{n}\right\}_{n=0}^{\infty}$ be a sequence in $(0, \infty)$ and $\left\{\alpha_{n}\right\}_{n=0}^{\infty},\left\{\beta_{n}\right\}_{n=0}^{\infty}$ be sequences in $[0,1]$ such that

$$
\liminf _{n \rightarrow \infty} r_{n}>0, \quad \limsup _{n \rightarrow \infty} \alpha_{n}<1, \quad \lim _{n \rightarrow \infty} \beta_{n}=1
$$

Let $\mathrm{EP} \cap F(S) \cap T^{-1} 0 \neq \emptyset$. If $S$ is uniformly continuous, then the sequence $\left\{x_{n}\right\}$ generated by Algorithm 3.1 converges strongly to $\prod_{\mathrm{EP} \cap F(S) \cap T^{-1} 0} x_{0}$.

Proof. First of all, if follows from the definition of $W_{n}$ that $x_{n}=\Pi_{W_{n}} x_{0}$. Since $x_{n+1}=$ $\Pi_{H_{n} \cap W_{n}} x_{0} \in W_{n}$, we have

$$
\phi\left(x_{n}, x_{0}\right) \leq \phi\left(x_{n+1}, x_{0}\right), \quad \forall n \geq 0 .
$$

Thus $\left\{\phi\left(x_{n}, x_{0}\right)\right\}$ is nondecreasing. Also from $x_{n}=\Pi_{W_{n}} x_{0}$ and Lemma 2.3, we have that

$$
\phi\left(x_{n}, x_{0}\right)=\phi\left(\Pi_{W_{n}} x_{0}, x_{0}\right) \leq \phi\left(w, x_{0}\right)-\phi\left(w, x_{n}\right) \leq \phi\left(w, x_{0}\right)
$$

for each $w \in \operatorname{EP} \cap F(S) \cap T^{-1} 0 \subset W_{n}$ and for each $n \geq 0$. Consequently, $\left\{\phi\left(x_{n}, x_{0}\right)\right\}$ is bounded. Moreover, according to the inequality

$$
\left(\left\|x_{n}\right\|-\left\|x_{0}\right\|\right)^{2} \leq \phi\left(x_{n}, x_{0}\right) \leq\left(\left\|x_{n}\right\|+\left\|x_{0}\right\|\right)^{2},
$$


we conclude that $\left\{x_{n}\right\}$ is bounded. Thus, we have that $\lim _{n \rightarrow \infty} \phi\left(x_{n}, x_{0}\right)$ exists. From Lemma 2.3, we derive the following:

$$
\begin{aligned}
\phi\left(x_{n+1}, x_{n}\right) & =\phi\left(x_{n+1}, \Pi_{W_{n}} x_{0}\right) \\
& \leq \phi\left(x_{n+1}, x_{0}\right)-\phi\left(\Pi_{W_{n}} x_{0}, x_{0}\right) \\
& =\phi\left(x_{n+1}, x_{0}\right)-\phi\left(x_{n}, x_{0}\right),
\end{aligned}
$$

for all $n \geq 0$. This implies that $\phi\left(x_{n+1}, x_{n}\right) \rightarrow 0$. So it follows from Lemma 2.1 that $x_{n+1}-x_{n} \rightarrow$ 0 . Since $x_{n+1}=\Pi_{H_{n} \cap W_{n}} x_{0} \in H_{n}$, from the definition of $H_{n}$, we also have

$$
\phi\left(x_{n+1}, u_{n}\right) \leq \alpha_{n} \phi\left(x_{n+1}, \tilde{x}_{n}\right)+\left(1-\alpha_{n}\right) \phi\left(x_{n+1}, z_{n}\right), \quad\left\langle x_{n+1}-\tilde{x}_{n}, v_{n}\right\rangle \leq 0 .
$$

Observe that

$$
\begin{aligned}
\phi\left(x_{n+1}, z_{n}\right) & =\phi\left(x_{n+1}, J^{-1}\left(\beta_{n} J \tilde{x}_{n}+\left(1-\beta_{n}\right) J S \tilde{x}_{n}\right)\right) \\
& =\left\|x_{n+1}\right\|^{2}-2\left\langle x_{n+1}, \beta_{n} J \tilde{x}_{n}+\left(1-\beta_{n}\right) J S \tilde{x}_{n}\right\rangle+\left\|\beta_{n} J \tilde{x}_{n}+\left(1-\beta_{n}\right) J S \tilde{x}_{n}\right\|^{2} \\
& \leq\left\|x_{n+1}\right\|^{2}-2 \beta_{n}\left\langle x_{n+1}, J \tilde{x}_{n}\right\rangle-2\left(1-\beta_{n}\right)\left\langle x_{n+1}, J S \tilde{x}_{n}\right\rangle+\beta_{n}\left\|\tilde{x}_{n}\right\|^{2}+\left(1-\beta_{n}\right)\left\|S \tilde{x}_{n}\right\|^{2} \\
& =\beta_{n} \phi\left(x_{n+1}, \tilde{x}_{n}\right)+\left(1-\beta_{n}\right) \phi\left(x_{n+1}, S \tilde{x}_{n}\right) .
\end{aligned}
$$

At the same time,

$$
\begin{aligned}
\phi\left(\Pi_{H_{n}} x_{n}, x_{n}\right)-\phi\left(\tilde{x}_{n}, x_{n}\right) & =\left\|\Pi_{H_{n}} x_{n}\right\|^{2}-\left\|\tilde{x}_{n}\right\|^{2}+2\left\langle\tilde{x}_{n}-\Pi_{H_{n}} x_{n}, J x_{n}\right\rangle \\
& \geq 2\left\langle\Pi_{H_{n}} x_{n}-\tilde{x}_{n}, J \tilde{x}_{n}\right\rangle+2\left\langle\tilde{x}_{n}-\Pi_{H_{n}} x_{n}, J x_{n}\right\rangle \\
& =2\left\langle\tilde{x}_{n}-\Pi_{H_{n}} x_{n}, J x_{n}-J \tilde{x}_{n}\right\rangle .
\end{aligned}
$$

Since $\Pi_{H_{n}} x_{n} \in H_{n}$ and $v_{n}=\left(1 / r_{n}\right)\left(J x_{n}-J \tilde{x}_{n}\right)$, it follows that

$$
\left\langle\tilde{x}_{n}-\Pi_{H_{n}} x_{n}, J x_{n}-J \tilde{x}_{n}\right\rangle=r_{n}\left\langle\tilde{x}_{n}-\Pi_{H_{n}} x_{n}, v_{n}\right\rangle \geq 0
$$

and hence that $\phi\left(\Pi_{H_{n}} x_{n}, x_{n}\right) \geq \phi\left(\tilde{x}_{n}, x_{n}\right)$. Further, from $x_{n+1} \in H_{n}$, we have $\phi\left(x_{n+1}, x_{n}\right) \geq$ $\phi\left(\Pi_{H_{n}} x_{n}, x_{n}\right)$, which yields

$$
\phi\left(x_{n+1}, x_{n}\right) \geq \phi\left(\Pi_{H_{n}} x_{n}, x_{n}\right) \geq \phi\left(\tilde{x}_{n}, x_{n}\right) .
$$


Then it follows from $\phi\left(x_{n+1}, x_{n}\right) \rightarrow 0$ that $\phi\left(\tilde{x}_{n}, x_{n}\right) \rightarrow 0$. Hence it follows from Lemma 2.1 that $\tilde{x}_{n}-x_{n} \rightarrow 0$. Since from (3.15) we derive

$$
\begin{aligned}
& \phi\left(x_{n+1}, \tilde{x}_{n}\right)-\phi\left(\tilde{x}_{n}, x_{n}\right) \\
&=\left\|x_{n+1}\right\|^{2}-2\left\langle x_{n+1}, J \tilde{x}_{n}\right\rangle+\left\|\tilde{x}_{n}\right\|^{2}-\left(\left\|\tilde{x}_{n}\right\|^{2}-2\left\langle\tilde{x}_{n}, J x_{n}\right\rangle+\left\|x_{n}\right\|^{2}\right) \\
&=\left\|x_{n+1}\right\|^{2}-\left\|x_{n}\right\|^{2}-2\left\langle x_{n+1}, J \tilde{x}_{n}\right\rangle+2\left\langle\tilde{x}_{n}, J x_{n}\right\rangle \\
&=\left\|x_{n+1}\right\|^{2}-\left\|x_{n}\right\|^{2}-2\left\langle x_{n+1}-\tilde{x}_{n}, J \tilde{x}_{n}-J x_{n}\right\rangle \\
&-2\left\langle x_{n+1}-\tilde{x}_{n}, J x_{n}\right\rangle+2\left\langle\tilde{x}_{n}, J x_{n}-J \tilde{x}_{n}\right\rangle \\
&=\left(\left\|x_{n+1}\right\|-\left\|x_{n}\right\|\right)\left(\left\|x_{n+1}\right\|+\left\|x_{n}\right\|\right)+2 r_{n}\left\langle x_{n+1}-\tilde{x}_{n}, v_{n}\right\rangle-2\left\langle x_{n+1}-\tilde{x}_{n}, J x_{n}\right\rangle \\
&+2\left\langle\tilde{x}_{n}, J x_{n}-J \tilde{x}_{n}\right\rangle \\
& \leq\left\|x_{n+1}-x_{n}\right\|\left(\left\|x_{n+1}\right\|+\left\|x_{n}\right\|\right)+2\left\|x_{n+1}-\tilde{x}_{n}\right\|\left\|x_{n}\right\|+2\left\|\tilde{x}_{n}\right\|\left\|J x_{n}-J \tilde{x}_{n}\right\| \\
& \leq\left\|x_{n+1}-x_{n}\right\|\left(\left\|x_{n+1}\right\|+\left\|x_{n}\right\|\right)+2\left(\left\|x_{n+1}-x_{n}\right\|+\left\|x_{n}-\tilde{x}_{n}\right\|\right)\left\|x_{n}\right\|+2\left\|\tilde{x}_{n}\right\|\left\|J x_{n}-J \tilde{x}_{n}\right\|,
\end{aligned}
$$

we have

$$
\begin{aligned}
\phi\left(x_{n+1}, \tilde{x}_{n}\right) \leq & \phi\left(\tilde{x}_{n}, x_{n}\right)+\left\|x_{n+1}-x_{n}\right\|\left(\left\|x_{n+1}\right\|+\left\|x_{n}\right\|\right) \\
& +2\left(\left\|x_{n+1}-x_{n}\right\|+\left\|x_{n}-\tilde{x}_{n}\right\|\right)\left\|x_{n}\right\|+2\left\|\tilde{x}_{n}\right\|\left\|J x_{n}-J \tilde{x}_{n}\right\| .
\end{aligned}
$$

Thus, from $\phi\left(\tilde{x}_{n}, x_{n}\right) \rightarrow 0, x_{n}-\tilde{x}_{n} \rightarrow 0$, and $x_{n+1}-x_{n} \rightarrow 0$, we know that $\phi\left(x_{n+1}, \tilde{x}_{n}\right) \rightarrow 0$. Consequently from (3.16), $\phi\left(x_{n+1}, \tilde{x}_{n}\right) \rightarrow 0$, and $\beta_{n} \rightarrow 1$ it follows that

$$
\phi\left(x_{n+1}, z_{n}\right) \longrightarrow 0
$$

So it follows from (3.15), $\phi\left(x_{n+1}, \tilde{x}_{n}\right) \rightarrow 0$, and $\phi\left(x_{n+1}, z_{n}\right) \rightarrow 0$ that $\phi\left(x_{n+1}, u_{n}\right) \rightarrow 0$. Utilizing Lemma 2.1 we deduce that

$$
\lim _{n \rightarrow \infty}\left\|x_{n+1}-u_{n}\right\|=\lim _{n \rightarrow \infty}\left\|x_{n+1}-\tilde{x}_{n}\right\|=\lim _{n \rightarrow \infty}\left\|x_{n+1}-z_{n}\right\|=0
$$


Furthermore, for $u \in \mathrm{EP} \cap F(S) \cap T^{-1} 0$ arbitrarily fixed, it follows from Proposition 2.8 that

$$
\begin{aligned}
\phi\left(u_{n}, y_{n}\right)= & \phi\left(K_{r_{n}} y_{n}, y_{n}\right) \leq \phi\left(u, y_{n}\right)-\phi\left(u, K_{r_{n}} y_{n}\right) \\
= & \phi\left(u, J^{-1}\left(\alpha_{n} J \tilde{x}_{n}+\left(1-\alpha_{n}\right) J S z_{n}\right)\right)-\phi\left(u, u_{n}\right) \\
= & \|u\|^{2}-2\left\langle u, \alpha_{n} J \tilde{x}_{n}+\left(1-\alpha_{n}\right) J S z_{n}\right\rangle+\left\|\alpha_{n} J \tilde{x}_{n}+\left(1-\alpha_{n}\right) J S z_{n}\right\|^{2}-\phi\left(u, u_{n}\right) \\
\leq & \|u\|^{2}-2 \alpha_{n}\left\langle u, J \tilde{x}_{n}\right\rangle-2\left(1-\alpha_{n}\right)\left\langle u, J S z_{n}\right\rangle+\alpha_{n}\left\|\tilde{x}_{n}\right\|^{2}+\left(1-\alpha_{n}\right)\left\|S z_{n}\right\|^{2}-\phi\left(u, u_{n}\right) \\
= & \alpha_{n} \phi\left(u, \tilde{x}_{n}\right)+\left(1-\alpha_{n}\right) \phi\left(u, S z_{n}\right)-\phi\left(u, u_{n}\right) \\
\leq & \left(1-\alpha_{n}\right) \phi\left(u, z_{n}\right)+\alpha_{n} \phi\left(u, \tilde{x}_{n}\right)-\phi\left(u, u_{n}\right) \\
= & \left(1-\alpha_{n}\right) \phi\left(u, J^{-1}\left(\beta_{n} J \tilde{x}_{n}+\left(1-\beta_{n}\right) J S \tilde{x}_{n}\right)\right)+\alpha_{n} \phi\left(u, \tilde{x}_{n}\right)-\phi\left(u, u_{n}\right) \\
= & \left(1-\alpha_{n}\right)\left[\|u\|^{2}-2\left\langle u, \beta_{n} J \tilde{x}_{n}+\left(1-\beta_{n}\right) J S \tilde{x}_{n}\right\rangle+\left\|\beta_{n} J \tilde{x}_{n}+\left(1-\beta_{n}\right) J S \tilde{x}_{n}\right\|^{2}\right] \\
& +\alpha_{n} \phi\left(u, \tilde{x}_{n}\right)-\phi\left(u, u_{n}\right) \\
\leq & \left(1-\alpha_{n}\right)\left[\|u\|^{2}-2 \beta_{n}\left\langle u, J \tilde{x}_{n}\right\rangle-2\left(1-\beta_{n}\right)\left\langle u, J S \tilde{x}_{n}\right\rangle+\beta_{n}\left\|\tilde{x}_{n}\right\|^{2}+\left(1-\beta_{n}\right)\left\|S \tilde{x}_{n}\right\|^{2}\right] \\
& +\alpha_{n} \phi\left(u, \tilde{x}_{n}\right)-\phi\left(u, u_{n}\right) \\
= & \left(1-\alpha_{n}\right)\left[\beta_{n} \phi\left(u, \tilde{x}_{n}\right)+\left(1-\beta_{n}\right) \phi\left(u, S \tilde{x}_{n}\right)\right]+\alpha_{n} \phi\left(u, \tilde{x}_{n}\right)-\phi\left(u, u_{n}\right) \\
\leq & \left(1-\alpha_{n}\right)\left[\beta_{n} \phi\left(u, \tilde{x}_{n}\right)+\left(1-\beta_{n}\right) \phi\left(u, \tilde{x}_{n}\right)\right]+\alpha_{n} \phi\left(u, \tilde{x}_{n}\right)-\phi\left(u, u_{n}\right) \\
= & \left(1-\alpha_{n}\right) \phi\left(u, \tilde{x}_{n}\right)+\alpha_{n} \phi\left(u, \tilde{x}_{n}\right)-\phi\left(u, u_{n}\right) \\
= & \phi\left(u, \tilde{x}_{n}\right)-\phi\left(u, u_{n}\right) \\
= & \phi\left(u, \tilde{x}_{n}\right)-\phi\left(u, x_{n+1}\right)+\phi\left(u, x_{n+1}\right)-\phi\left(u, u_{n}\right) \\
= & \left\|\tilde{x}_{n}\right\|^{2}-\left\|x_{n+1}\right\|^{2}+2\left\langle u, J x_{n+1}-J \tilde{x}_{n}\right\rangle+\left\|x_{n+1}\right\|^{2}-\left\|u_{n}\right\|^{2}+2\left\langle u, J u_{n}-J x_{n+1}\right\rangle \\
\leq & \left\|\tilde{x}_{n}-x_{n+1}\right\|\left(\left\|\tilde{x}_{n}\right\|+\left\|x_{n+1}\right\|\right)+2\|u\|\left\|J x_{n+1}-J \tilde{x}_{n}\right\| \\
& +u_{n}\left\|\left(\left\|x_{n+1}\right\|+\left\|u_{n}\right\|\right)+2\right\| u\|\| J u_{n}-J x_{n+1} \| . \\
& \\
&
\end{aligned}
$$

Since $J$ is uniformly norm-to-norm continuous on bounded subsets of $E$, it follows from (3.23) that $\left\|J x_{n+1}-J \tilde{x}_{n}\right\| \rightarrow 0$ and $\left\|J u_{n}-J x_{n+1}\right\| \rightarrow 0$, which hence yield $\phi\left(u_{n}, y_{n}\right) \rightarrow 0$. Utilizing Lemma 2.1, we get $\left\|u_{n}-y_{n}\right\| \rightarrow 0$. Observe that

$$
\left\|x_{n+1}-y_{n}\right\| \leq\left\|x_{n+1}-u_{n}\right\|+\left\|u_{n}-y_{n}\right\| \longrightarrow 0,
$$

due to (3.23). Since $J$ is uniformly norm-to-norm continuous on bounded subsets of $E$, we have that

$$
\lim _{n \rightarrow \infty}\left\|J x_{n+1}-J y_{n}\right\|=\lim _{n \rightarrow \infty}\left\|J x_{n+1}-J \tilde{x}_{n}\right\|=0 .
$$


On the other hand, we have

$$
\left\|x_{n}-z_{n}\right\| \leq\left\|x_{n}-x_{n+1}\right\|+\left\|x_{n+1}-z_{n}\right\| \longrightarrow 0
$$

Noticing that

$$
\begin{aligned}
\left\|J x_{n+1}-J y_{n}\right\| & =\left\|J x_{n+1}-\left(\alpha_{n} J \tilde{x}_{n}+\left(1-\alpha_{n}\right) J S z_{n}\right)\right\| \\
& =\left\|\alpha_{n}\left(J x_{n+1}-J \tilde{x}_{n}\right)+\left(1-\alpha_{n}\right)\left(J x_{n+1}-J S z_{n}\right)\right\| \\
& =\left\|\left(1-\alpha_{n}\right)\left(J x_{n+1}-J S z_{n}\right)-\alpha_{n}\left(J \tilde{x}_{n}-J x_{n+1}\right)\right\| \\
& \geq\left(1-\alpha_{n}\right)\left\|J x_{n+1}-J S z_{n}\right\|-\alpha_{n}\left\|J \tilde{x}_{n}-J x_{n+1}\right\|,
\end{aligned}
$$

we have

$$
\left\|J x_{n+1}-J S z_{n}\right\| \leq \frac{1}{1-\alpha_{n}}\left(\left\|J x_{n+1}-J y_{n}\right\|+\alpha_{n}\left\|J \tilde{x}_{n}-J x_{n+1}\right\|\right)
$$

From (3.26) and $\lim \sup _{n \rightarrow \infty} \alpha_{n}<1$, we obtain

$$
\lim _{n \rightarrow \infty}\left\|J x_{n+1}-J S z_{n}\right\|=0 .
$$

Since $J^{-1}$ is also uniformly norm-to-norm continuous on bounded subsets of $E^{*}$, we obtain

$$
\lim _{n \rightarrow \infty}\left\|x_{n+1}-S z_{n}\right\|=0
$$

Observe that

$$
\left\|x_{n}-S x_{n}\right\| \leq\left\|x_{n}-x_{n+1}\right\|+\left\|x_{n+1}-S z_{n}\right\|+\left\|S z_{n}-S x_{n}\right\| .
$$

Since $S$ is uniformly continuous, it follows from (3.27), (3.31) and $x_{n+1}-x_{n} \rightarrow 0$ that $x_{n}-$ $S x_{n} \rightarrow 0$.

Now let us show that $\omega_{w}\left(\left\{x_{n}\right\}\right) \subset \mathrm{EP} \cap F(S) \cap T^{-1} 0$, where

$$
\omega_{w}\left(\left\{x_{n}\right\}\right):=\left\{\widehat{x} \in C: x_{n_{k}} \rightarrow \widehat{x} \text { for some subsequence }\left\{n_{k}\right\} \subset\{n\} \text { with } n_{k} \uparrow \infty\right\} \text {. }
$$

Indeed, since $\left\{x_{n}\right\}$ is bounded and $X$ is reflexive, we know that $\omega_{w}\left(\left\{x_{n}\right\}\right) \neq \emptyset$. Take $\hat{x} \in$ $\omega_{w}\left(\left\{x_{n}\right\}\right)$ arbitrarily, then there exists a subsequence $\left\{x_{n_{k}}\right\}$ of $\left\{x_{n}\right\}$ such that $x_{n_{k}}-\widehat{x}$. Hence $\hat{x} \in F(S)$. Let us show that $\hat{x} \in T^{-1} 0$. Since $x_{n}-\widetilde{x}_{n} \rightarrow 0$, we have that $\tilde{x}_{n_{k}} \rightarrow \widehat{x}$. Moreover, since $J$ is uniformly norm-to-norm continuous on bounded subsets of $E$ and $\lim \inf _{n \rightarrow \infty} r_{n}>0$, we obtain

$$
v_{n}=\frac{1}{r_{n}}\left(J x_{n}-J \tilde{x}_{n}\right) \longrightarrow 0 .
$$


It follows from $v_{n} \in T \tilde{x}_{n}$ and the monotonicity of $T$ that

$$
\left\langle z-\tilde{x}_{n}, z^{\prime}-v_{n}\right\rangle \geq 0
$$

for all $z \in D(T)$ and $z^{\prime} \in T z$. This implies that

$$
\left\langle z-\widehat{x}, z^{\prime}\right\rangle \geq 0
$$

for all $z \in D(T)$ and $z^{\prime} \in T z$. Thus from the maximality of $T$, we infer that $\hat{x} \in T^{-1} 0$. Therefore, $\hat{x} \in F(S) \cap T^{-1} 0$. Further, let us show that $\hat{x} \in$ EP. Since $u_{n}-y_{n} \rightarrow 0$ and $x_{n}-u_{n} \rightarrow 0$, from $x_{n_{k}} \rightarrow \widehat{x}$ we obtain that $y_{n_{k}} \rightarrow \widehat{x}$ and $u_{n_{k}} \rightarrow \widehat{x}$.

Since $J$ is uniformly norm-to-norm continuous on bounded subsets of $E$, from $u_{n}$ $y_{n} \rightarrow 0$ we derive

$$
\lim _{n \rightarrow \infty}\left\|J u_{n}-J y_{n}\right\|=0
$$

From $\lim \inf _{n \rightarrow \infty} r_{n}>0$, it follows that

$$
\lim _{n \rightarrow \infty} \frac{\left\|J u_{n}-J y_{n}\right\|}{r_{n}}=0 .
$$

By the definition of $u_{n}:=K_{r_{n}} y_{n}$, we have

$$
F\left(u_{n}, y\right)+\frac{1}{r_{n}}\left\langle y-u_{n}, J u_{n}-J y_{n}\right\rangle \geq 0, \quad \forall y \in C,
$$

where

$$
F\left(u_{n}, y\right)=f\left(u_{n}, y\right)+\left\langle A u_{n}, y-u_{n}\right\rangle
$$

Replacing $n$ by $n_{k}$, we have from (A2) that

$$
\frac{1}{r_{n_{k}}}\left\langle y-u_{n_{k}}, J u_{n_{k}}-J y_{n_{k}}\right\rangle \geq-F\left(u_{n_{k}}, y\right) \geq F\left(y, u_{n_{k}}\right), \quad \forall y \in C .
$$

Since $y \mapsto f(x, y)+\langle A x, y-x\rangle$ is convex and lower semicontinuous, it is also weakly lower semicontinuous. Letting $n_{k} \rightarrow \infty$ in the last inequality, from (3.38) and (A4) we have

$$
F(y, \widehat{x}) \leq 0, \quad \forall y \in C
$$

For $t$, with $0<t \leq 1$, and $y \in C$, let $y_{t}=t y+(1-t) \widehat{x}$. Since $y \in C$ and $\widehat{x} \in C$, then $y_{t} \in C$ and hence $F\left(y_{t}, \widehat{x}\right) \leq 0$. So, from (A1) we have

$$
0=F\left(y_{t}, y_{t}\right) \leq t F\left(y_{t}, y\right)+(1-t) F\left(y_{t}, \widehat{x}\right) \leq t F\left(y_{t}, y\right)
$$


Dividing by $t$, we have

$$
F\left(y_{t}, y\right) \geq 0, \quad \forall y \in C
$$

Letting $t \downarrow 0$, from (A3) it follows that

$$
F(\widehat{x}, y) \geq 0, \quad \forall y \in C
$$

So, $\widehat{x} \in$ EP. Therefore, we obtain that $\omega_{w}\left(\left\{x_{n}\right\}\right) \subset \mathrm{EP} \cap F(S) \cap T^{-1} 0$ by the arbitrariness of $\widehat{x}$.

Next, let us show that $\omega_{w}\left(\left\{x_{n}\right\}\right)=\left\{\prod_{\mathrm{EP} \cap F(S) \cap T^{-1} 0} x_{0}\right\}$ and $x_{n} \rightarrow \prod_{\mathrm{EP} \cap F(S) \cap T^{-1} 0} x_{0}$.

Indeed, put $\bar{x}=\Pi_{\mathrm{EP} \cap F(S) \cap T^{-1} 0} x_{0}$. From $x_{n+1}=\Pi_{H_{n} \cap W_{n}} x_{0}$ and $\bar{x} \in \operatorname{EP} \cap F(S) \cap T^{-1} 0 \subset$ $H_{n} \cap W_{n}$, we have $\phi\left(x_{n+1}, x_{0}\right) \leq \phi\left(\bar{x}, x_{0}\right)$. Now from weakly lower semicontinuity of the norm, we derive for each $\widehat{x} \in \omega_{w}\left(\left\{x_{n}\right\}\right)$

$$
\begin{aligned}
\phi\left(\widehat{x}, x_{0}\right) & =\|\widehat{x}\|^{2}-2\left\langle\widehat{x}, x_{0}\right\rangle+\left\|x_{0}\right\|^{2} \\
& \leq \liminf _{k \rightarrow \infty}\left(\left\|x_{n_{k}}\right\|^{2}-2\left\langle x_{n_{k}}, x_{0}\right\rangle+\left\|x_{0}\right\|^{2}\right) \\
& =\liminf _{k \rightarrow \infty} \phi\left(x_{n_{k}}, x_{0}\right) \\
& \leq \limsup _{k \rightarrow \infty} \phi\left(x_{n_{k}}, x_{0}\right) \\
& \leq \phi\left(\bar{x}, x_{0}\right) .
\end{aligned}
$$

It follows from the definition of $\prod_{\mathrm{EP} \cap F(S) \cap T^{-1} 0} x_{0}$ that $\widehat{x}=\bar{x}$ and hence

$$
\lim _{k \rightarrow \infty} \phi\left(x_{n_{k}}, x_{0}\right)=\phi\left(\bar{x}, x_{0}\right)
$$

So we have $\lim _{k \rightarrow \infty}\left\|x_{n_{k}}\right\|=\|\bar{x}\|$. Utilizing the Kadec-Klee property of $E$, we conclude that $\left\{x_{n_{k}}\right\}$ converges strongly to $\Pi_{\mathrm{EP} \cap F(S) \cap T^{-1} 0} x_{0}$. Since $\left\{x_{n_{k}}\right\}$ is an arbitrary weakly convergent subsequence of $\left\{x_{n}\right\}$, we know that $\left\{x_{n}\right\}$ converges strongly to $\prod_{\operatorname{EP} \cap F(S) \cap T^{-1} 0} x_{0}$. This completes the proof.

Theorem 3.5 covers [25, Theorem 3.1] by taking $C=E, f \equiv 0$ and $A \equiv 0$. Also Theorem 3.5 covers [24, Theorem 2.1] by taking $f \equiv 0, A \equiv 0$ and $T \equiv 0$.

Theorem 3.6. Let $C$ be a nonempty closed convex subset of a uniformly smooth and uniformly convex Banach space $E$. Let $T: E \rightarrow 2^{E^{*}}$ be a maximal monotone operator with domain $D(T)=C, S: C \rightarrow$ $C$ be a relatively nonexpansive mapping, $A: C \rightarrow E^{*}$ be an $\alpha$-inverse-strongly monotone mapping and $f: C \times C \rightarrow R$ be a bifunction satisfying $(A 1)-(A 4)$. Assume that $\left\{r_{n}\right\}_{n=0}^{\infty}$ is a sequence in $(0, \infty)$ satisfying $\liminf _{n \rightarrow \infty} r_{n}>0$ and that $\left\{\alpha_{n}\right\}_{n=0}^{\infty}$ is a sequences in $(0,1)$ satisfying $\lim _{n \rightarrow \infty} \alpha_{n}=0$.

Define a sequence $\left\{x_{n}\right\}$ by the following algorithm. 
Algorithm 3.7.

$$
\begin{gathered}
x_{0} \in C \text { arbitrarily chosen, } \\
0=v_{n}+\frac{1}{r_{n}}\left(J \tilde{x}_{n}-J x_{n}\right), \quad v_{n} \in T \tilde{x}_{n}, \\
y_{n}=J^{-1}\left(\alpha_{n} J x_{0}+\left(1-\alpha_{n}\right) J S \tilde{x}_{n}\right), \\
u_{n} \in C \text { such that } \\
f\left(u_{n}, y\right)+\left\langle A u_{n}, y-u_{n}\right\rangle+\frac{1}{r_{n}}\left\langle y-u_{n}, J u_{n}-J y_{n}\right\rangle \geq 0, \quad \forall y \in C, \\
H_{n}=\left\{v \in C: \phi\left(v, u_{n}\right) \leq \alpha_{n} \phi\left(v, x_{0}\right)+\left(1-\alpha_{n}\right) \phi\left(v, \tilde{x}_{n}\right),\left\langle v-\tilde{x}_{n}, v_{n}\right\rangle \leq 0\right\}, \\
W_{n}=\left\{v \in C:\left\langle v-x_{n}, J x_{0}-J x_{n}\right\rangle \leq 0\right\}, \\
x_{n+1}=\Pi_{H_{n} \cap W_{n}} x_{0}, \quad n=0,1,2, \ldots,
\end{gathered}
$$

where $J$ is the single valued duality mapping on $E$. Let $\operatorname{EP} \cap F(S) \cap T^{-1} 0 \neq \emptyset$. If $S$ is uniformly continuous, then $\left\{x_{n}\right\}$ converges strongly to $\prod_{\mathrm{EP} \cap F(S) \cap T^{-1} 0} x_{0}$.

Proof. For each $n \geq 0$, define two sets $C_{n}$ and $D_{n}$ as follows:

$$
\begin{gathered}
C_{n}=\left\{v \in C: \phi\left(v, u_{n}\right) \leq \alpha_{n} \phi\left(v, x_{0}\right)+\left(1-\alpha_{n}\right) \phi\left(v, \tilde{x}_{n}\right)\right\}, \\
D_{n}=\left\{v \in C:\left\langle v-\tilde{x}_{n}, v_{n}\right\rangle \leq 0\right\} .
\end{gathered}
$$

It is obvious that $C_{n}$ is closed and $D_{n}, W_{n}$ are closed convex sets for each $n \geq 0$. Let us show that $C_{n}$ is convex and so $H_{n}=C_{n} \cap D_{n}$ is closed and convex. Similarly to the proof of Lemma 3.3, since

$$
\phi\left(v, u_{n}\right) \leq \alpha_{n} \phi\left(v, x_{0}\right)+\left(1-\alpha_{n}\right) \phi\left(v, \tilde{x}_{n}\right)
$$

is equivalent to

$$
2 \alpha_{n}\left\langle v, J x_{0}\right\rangle+2\left(1-\alpha_{n}\right)\left\langle v, J \tilde{x}_{n}\right\rangle-2\left\langle v, J u_{n}\right\rangle \leq \alpha_{n}\left\|x_{0}\right\|^{2}+\left(1-\alpha_{n}\right)\left\|\tilde{x}_{n}\right\|^{2}-\left\|u_{n}\right\|^{2},
$$


we know that $C_{n}$ is convex and so is $H_{n}=C_{n} \cap D_{n}$. Next, let us show that $\mathrm{EP} \cap F(S) \cap T^{-1} 0 \subset$ $C_{n}$ for each $n \geq 0$. Indeed, utilizing Proposition 2.8, we have, for each $w \in \operatorname{EP} \cap F(S) \cap T^{-1} 0$,

$$
\begin{aligned}
\phi\left(w, u_{n}\right) & =\phi\left(w, K_{r_{n}} y_{n}\right) \leq \phi\left(w, y_{n}\right) \\
& =\phi\left(w, J^{-1}\left(\alpha_{n} J x_{0}+\left(1-\alpha_{n}\right) J S \tilde{x}_{n}\right)\right) \\
& =\|w\|^{2}-2\left\langle w, \alpha_{n} J x_{0}+\left(1-\alpha_{n}\right) J S \tilde{x}_{n}\right\rangle+\left\|\alpha_{n} J x_{0}+\left(1-\alpha_{n}\right) J S \tilde{x}_{n}\right\|^{2} \\
& \leq\|w\|^{2}-2 \alpha_{n}\left\langle w, J x_{0}\right\rangle-2\left(1-\alpha_{n}\right)\left\langle w, J S \tilde{x}_{n}\right\rangle+\alpha_{n}\left\|x_{0}\right\|^{2}+\left(1-\alpha_{n}\right)\left\|S \tilde{x}_{n}\right\|^{2} \\
& =\alpha_{n} \phi\left(w, x_{0}\right)+\left(1-\alpha_{n}\right) \phi\left(w, S \tilde{x}_{n}\right) \\
& \leq \alpha_{n} \phi\left(w, x_{0}\right)+\left(1-\alpha_{n}\right) \phi\left(w, \tilde{x}_{n}\right) .
\end{aligned}
$$

So $w \in C_{n}$ for all $n \geq 0$ and $\mathrm{EP} \cap F(S) \cap T^{-1} 0 \subset C_{n}$. As in the proof of Lemma 3.3, we can obtain $w \in D_{n}$ and hence $w \in H_{n}$. It follows from Lemma 2.4 that

$$
\left\langle w-x_{n}, J x_{0}-J x_{n}\right\rangle=\left\langle w-\Pi_{H_{n-1} \cap W_{n-1}} x_{0}, J x_{0}-J \Pi_{H_{n-1} \cap W_{n-1}} x_{0}\right\rangle \leq 0,
$$

which implies that $w \in W_{n}$. Consequently, $w \in H_{n} \cap W_{n}$ and so $\mathrm{EP} \cap F(S) \cap T^{-1} 0 \subset H_{n} \cap W_{n}$ for all $n \geq 0$. Therefore, the sequence $\left\{x_{n}\right\}$ generated by Algorithm 3.7 is well defined. As in the proof of Theorem 3.5, we can obtain $\phi\left(x_{n+1}, x_{n}\right) \rightarrow 0$. Since $x_{n+1}=\Pi_{H_{n} \cap W_{n}} x_{0} \in H_{n}$, from the definition of $H_{n}$ we also have

$$
\phi\left(x_{n+1}, u_{n}\right) \leq \alpha_{n} \phi\left(x_{n+1}, x_{0}\right)+\left(1-\alpha_{n}\right) \phi\left(x_{n+1}, \tilde{x}_{n}\right), \quad\left\langle x_{n+1}-\tilde{x}_{n}, v_{n}\right\rangle \leq 0 .
$$

As in the proof of Theorem 3.5, we can deduce not only from $\phi\left(x_{n+1}, x_{n}\right) \rightarrow 0$ that $\phi\left(\tilde{x}_{n}, x_{n}\right) \rightarrow 0$ but also from $\phi\left(\tilde{x}_{n}, x_{n}\right) \rightarrow 0, x_{n}-\tilde{x}_{n} \rightarrow 0$ and $x_{n+1}-x_{n} \rightarrow 0$ that

$$
\lim _{n \rightarrow \infty} \phi\left(x_{n+1}, \tilde{x}_{n}\right)=0
$$

Since $x_{n+1}=\Pi_{H_{n} \cap W_{n}} x_{0} \in H_{n}$, from the definition of $H_{n}$, we also have

$$
\phi\left(x_{n+1}, u_{n}\right) \leq \alpha_{n} \phi\left(x_{n+1}, x_{0}\right)+\left(1-\alpha_{n}\right) \phi\left(x_{n+1}, \tilde{x}_{n}\right) .
$$

It follows from (3.55) and $\alpha_{n} \rightarrow 0$ that

$$
\lim _{n \rightarrow \infty} \phi\left(x_{n+1}, u_{n}\right)=0
$$

Utilizing Lemma 2.1 we have

$$
\lim _{n \rightarrow \infty}\left\|x_{n+1}-u_{n}\right\|=\lim _{n \rightarrow \infty}\left\|x_{n+1}-x_{n}\right\|=\lim _{n \rightarrow \infty}\left\|x_{n+1}-\tilde{x}_{n}\right\|=0 .
$$


Furthermore, for $u \in E P \cap F(S) \cap T^{-1} 0$ arbitrarily fixed, it follows from Proposition 2.8 that

$$
\begin{aligned}
\phi\left(u_{n}, y_{n}\right)= & \phi\left(K_{r_{n}} y_{n}, y_{n}\right) \leq \phi\left(u, y_{n}\right)-\phi\left(u, K_{r_{n}} y_{n}\right) \\
= & \phi\left(u, J^{-1}\left(\alpha_{n} J x_{0}+\left(1-\alpha_{n}\right) J S \tilde{x}_{n}\right)\right)-\phi\left(u, u_{n}\right) \\
= & \|u\|^{2}-2\left\langle u, \alpha_{n} J x_{0}+\left(1-\alpha_{n}\right) J S \tilde{x}_{n}\right\rangle+\left\|\alpha_{n} J x_{0}+\left(1-\alpha_{n}\right) J S \tilde{x}_{n}\right\|^{2}-\phi\left(u, u_{n}\right) \\
\leq & \|u\|^{2}-2 \alpha_{n}\left\langle u, J x_{0}\right\rangle-2\left(1-\alpha_{n}\right)\left\langle u, J S \tilde{x}_{n}\right\rangle+\alpha_{n}\left\|x_{0}\right\|^{2}+\left(1-\alpha_{n}\right)\left\|S \tilde{x}_{n}\right\|^{2}-\phi\left(u, u_{n}\right) \\
= & \alpha_{n} \phi\left(u, x_{0}\right)+\left(1-\alpha_{n}\right) \phi\left(u, S \tilde{x}_{n}\right)-\phi\left(u, u_{n}\right) \\
\leq & \alpha_{n} \phi\left(u, x_{0}\right)+\phi\left(u, \tilde{x}_{n}\right)-\phi\left(u, u_{n}\right) \\
= & \alpha_{n} \phi\left(u, x_{0}\right)+\phi\left(u, \tilde{x}_{n}\right)-\phi\left(u, x_{n+1}\right)+\phi\left(u, x_{n+1}\right)-\phi\left(u, u_{n}\right) \\
= & \alpha_{n} \phi\left(u, x_{0}\right)+\left\|\tilde{x}_{n}\right\|^{2}-\left\|x_{n+1}\right\|^{2}+2\left\langle u, J x_{n+1}-J \tilde{x}_{n}\right\rangle+\left\|x_{n+1}\right\|^{2} \\
& -\left\|u_{n}\right\|^{2}+2\left\langle u, J u_{n}-J x_{n+1}\right\rangle \\
\leq & \alpha_{n} \phi\left(u, x_{0}\right)+\left\|\tilde{x}_{n}-x_{n+1}\right\|\left(\left\|\tilde{x}_{n}\right\|+\left\|x_{n+1}\right\|\right)+2\|u\|\left\|J x_{n+1}-J \tilde{x}_{n}\right\| \\
& +\left\|x_{n+1}-u_{n}\right\|\left(\left\|x_{n+1}\right\|+\left\|u_{n}\right\|\right)+2\|u\|\left\|J u_{n}-J x_{n+1}\right\| .
\end{aligned}
$$

Since $J$ is uniformly norm-to-norm continuous on bounded subsets of $E$, it follows from (3.58) that $\left\|J x_{n+1}-J \tilde{x}_{n}\right\| \rightarrow 0$ and $\left\|J u_{n}-J x_{n+1}\right\| \rightarrow 0$, which together with $\alpha_{n} \rightarrow 0$, yield $\phi\left(u_{n}, y_{n}\right) \rightarrow 0$. Utilizing Lemma 2.1 , we get $\left\|u_{n}-y_{n}\right\| \rightarrow 0$. Observe that

$$
\left\|x_{n+1}-y_{n}\right\| \leq\left\|x_{n+1}-u_{n}\right\|+\left\|u_{n}-y_{n}\right\| \longrightarrow 0,
$$

due to (3.58). Since $J$ is uniformly norm-to-norm continuous on bounded subsets of $E$, we have

$$
\lim _{n \rightarrow \infty}\left\|J x_{n+1}-J y_{n}\right\|=\lim _{n \rightarrow \infty}\left\|J x_{n+1}-J x_{n}\right\|=\lim _{n \rightarrow \infty}\left\|J x_{n+1}-J \tilde{x}_{n}\right\|=0
$$

Note that

$$
\left\|J S \tilde{x}_{n}-J y_{n}\right\|=\left\|J S \tilde{x}_{n}-\left(\alpha_{n} J x_{0}+\left(1-\alpha_{n}\right) J S \tilde{x}_{n}\right)\right\|=\alpha_{n}\left\|J x_{0}-J S \tilde{x}_{n}\right\| .
$$

Therefore, from $\alpha_{n} \rightarrow 0$ we get

$$
\lim _{n \rightarrow \infty}\left\|J S \tilde{x}_{n}-J y_{n}\right\|=0
$$

Since $J^{-1}$ is also uniformly norm-to-norm continuous on bounded subsets of $E^{*}$, we obtain

$$
\lim _{n \rightarrow \infty}\left\|S \widetilde{x}_{n}-y_{n}\right\|=0
$$


It follows that

$$
\left\|x_{n}-S x_{n}\right\| \leq\left\|x_{n}-x_{n+1}\right\|+\left\|x_{n+1}-y_{n}\right\|+\left\|y_{n}-S \tilde{x}_{n}\right\|+\left\|S \tilde{x}_{n}-S x_{n}\right\|
$$

Since $S$ is uniformly continuous, it follows from (3.58) and (3.64) that $x_{n}-S x_{n} \rightarrow 0$.

Finally, we prove that $x_{n} \rightarrow \Pi_{\mathrm{EP} \cap F(S) \cap T^{-1} 0} x_{0}$. Indeed, for $\hat{x} \in \mathrm{EP} \cap F(S) \cap T^{-1} 0$ arbitrarily fixed, there exists a subsequence $\left\{x_{n_{k}}\right\}$ of $\left\{x_{n}\right\}$ such that $x_{n_{k}} \rightarrow \hat{x} \in C$, then $\hat{x} \in F(S)$. Now let us show that $\hat{x} \in T^{-1} 0$. Since $x_{n}-\tilde{x}_{n} \rightarrow 0$, we have that $\tilde{x}_{n_{k}} \rightarrow \hat{x}$. Moreover, since $J$ is uniformly norm-to-norm continuous on bounded subsets of $E$, and $\liminf \operatorname{in}_{n \rightarrow \infty} r_{n}>0$, we obtain that $v_{n}=\left(1 / r_{n}\right)\left(J x_{n}-J \tilde{x}_{n}\right) \rightarrow 0$. It follows from $v_{n} \in T \tilde{x}_{n}$ and the monotonicity of $T$ that $\left\langle z-\tilde{x}_{n}, z^{\prime}-v_{n}\right\rangle \geq 0$ for all $z \in D(T)$ and $z^{\prime} \in T z$. This implies that $\left\langle z-\hat{x}, z^{\prime}\right\rangle \geq 0$ for all $z \in D(T)$ and $z^{\prime} \in T z$. Thus from the maximality of $T$, we infer that $\hat{x} \in T^{-1} 0$. Further, let us show that $\widehat{x} \in \mathrm{EP}$. Since $u_{n}-y_{n} \rightarrow 0$ and $x_{n}-u_{n} \rightarrow 0$, from $x_{n_{k}}-\widehat{x}$ we obtain that $y_{n_{k}} \rightarrow \widehat{x}$ and $u_{n_{k}} \rightarrow \widehat{x}$.

Since $J$ is uniformly norm-to-norm continuous on bounded subsets of $E$, from $u_{n}$ $y_{n} \rightarrow 0$ we derive $\lim _{n \rightarrow \infty}\left\|J u_{n}-J y_{n}\right\|=0$. From $\liminf _{n \rightarrow \infty} r_{n}>0$ it follows that

$$
\lim _{n \rightarrow \infty} \frac{\left\|J u_{n}-J y_{n}\right\|}{r_{n}}=0
$$

By the definition of $u_{n}:=K_{r_{n}} y_{n}$, we have

$$
F\left(u_{n}, y\right)+\frac{1}{r_{n}}\left\langle y-u_{n}, J u_{n}-J y_{n}\right\rangle \geq 0, \quad \forall y \in C,
$$

where $F\left(u_{n}, y\right)=f\left(u_{n}, y\right)+\left\langle A u_{n}, y-u_{n}\right\rangle$. Replacing $n$ by $n_{k}$, we have from (A2) that

$$
\frac{1}{r_{n_{k}}}\left\langle y-u_{n_{k}}, J u_{n_{k}}-J y_{n_{k}}\right\rangle \geq-F\left(u_{n_{k}}, y\right) \geq F\left(y, u_{n_{k}}\right), \quad \forall y \in C
$$

Since $y \mapsto f(x, y)+\langle A x, y-x\rangle$ is convex and lower semicontinuous, it is also weakly lower semicontinuous. Letting $n_{k} \rightarrow \infty$ in the last inequality, from (3.66) and (A4) we have $F(y, \widehat{x}) \leq 0$, for all $y \in C$. For $t$, with $0<t \leq 1$, and $y \in C$, let $y_{t}=t y+(1-t) \widehat{x}$. Since $y \in C$ and $\hat{x} \in C$, then $y_{t} \in C$ and hence $F\left(y_{t}, \widehat{x}\right) \leq 0$. So, from (A1) we have

$$
0=F\left(y_{t}, y_{t}\right) \leq t F\left(y_{t}, y\right)+(1-t) F\left(y_{t}, \widehat{x}\right) \leq t F\left(y_{t}, y\right)
$$

Dividing by $t$, we have $F\left(y_{t}, y\right) \geq 0$, for all $y \in C$. Letting $t \downarrow 0$, from (A3) it follows that $F(\widehat{x}, y) \geq 0$, for all $y \in C$. So, $\widehat{x} \in \mathrm{EP}$. Therefore, we obtain that $\omega_{w}\left(\left\{x_{n}\right\}\right) \subset \mathrm{EP} \cap F(S) \cap T^{-1} 0$ by the arbitrariness of $\hat{x}$.

Next, let us show that $\omega_{w}\left(\left\{x_{n}\right\}\right)=\left\{\prod_{\mathrm{EP} \cap F(S) \cap T^{-1} 0} x_{0}\right\}$ and $x_{n} \rightarrow \prod_{\mathrm{EP} \cap F(S) \cap T^{-1} 0} x_{0}$. 
Indeed, put $\bar{x}=\Pi_{\mathrm{EP} \cap F(S) \cap T^{-1} 0} x_{0}$. From $x_{n+1}=\Pi_{H_{n} \cap W_{n}} x_{0}$ and $\bar{x} \in \mathrm{EP} \cap F(S) \cap T^{-1} 0 \subset$ $H_{n} \cap W_{n}$, we have $\phi\left(x_{n+1}, x_{0}\right) \leq \phi\left(\bar{x}, x_{0}\right)$. Now from weakly lower semicontinuity of the norm, we derive for each $\widehat{x} \in \omega_{w}\left(\left\{x_{n}\right\}\right)$

$$
\begin{aligned}
\phi\left(\widehat{x}, x_{0}\right) & =\|\widehat{x}\|^{2}-2\left\langle\widehat{x}, x_{0}\right\rangle+\left\|x_{0}\right\|^{2} \\
& \leq \liminf _{k \rightarrow \infty}\left(\left\|x_{n_{k}}\right\|^{2}-2\left\langle x_{n_{k}}, x_{0}\right\rangle+\left\|x_{0}\right\|^{2}\right) \\
& =\liminf _{k \rightarrow \infty} \phi\left(x_{n_{k}}, x_{0}\right) \\
& \leq \limsup _{k \rightarrow \infty} \phi\left(x_{n_{k}}, x_{0}\right) \\
& \leq \phi\left(\bar{x}, x_{0}\right) .
\end{aligned}
$$

It follows from the definition of $\Pi_{\operatorname{EP} \cap F(S) \cap T^{-1} 0} x_{0}$ that $\widehat{x}=\bar{x}$ and hence $\lim _{k \rightarrow \infty} \phi\left(x_{n_{k}}, x_{0}\right)=$ $\phi\left(\bar{x}, x_{0}\right)$. So we have $\lim _{k \rightarrow \infty}\left\|x_{n_{k}}\right\|=\|\bar{x}\|$. Utilizing the Kadec-Klee property of $E$, we know that $x_{n_{k}} \rightarrow \prod_{\mathrm{EP} \cap F(S) \cap T^{-1} 0} x_{0}$. Since $\left\{x_{n_{k}}\right\}$ is an arbitrary weakly convergent subsequence of $\left\{x_{n}\right\}$, we know that $x_{n} \rightarrow \prod_{\mathrm{EP} \cap F(S) \cap T^{-1} 0} x_{0}$. This completes the proof.

Theorem 3.6 covers [25, Theorem 3.2] by taking $C=E, f \equiv 0$ and $A \equiv 0$. Also Theorem 3.6 covers [24, Theorem 2.2] by taking $f \equiv 0, A \equiv 0$ and $T \equiv 0$.

\section{Acknowledgments}

This research was partially supported by the Leading Academic Discipline Project of Shanghai Normal University (no. DZL707), Innovation Program of Shanghai Municipal Education Commission Grant (no. 09ZZ133), National Science Foundation of China (no. 11071169), Ph.D. Program Foundation of Ministry of Education of China (no. 20070270004), Science and Technology Commission of Shanghai Municipality Grant (no. 075105118), and Shanghai Leading Academic Discipline Project (no. S30405). Al-Homidan is grateful to KFUPM for providing research facilities.

\section{References}

[1] S. Takahashi and W. Takahashi, "Strong convergence theorem for a generalized equilibrium problem and a nonexpansive mapping in a Hilbert space," Nonlinear Analysis. Theory, Methods \& Applications, vol. 69, no. 3, pp. 1025-1033, 2008.

[2] L.-C. Zeng and J.-C. Yao, "Strong convergence theorem by an extragradient method for fixed point problems and variational inequality problems," Taiwanese Journal of Mathematics, vol. 10, no. 5, pp. 1293-1303, 2006.

[3] S. Schaible, J.-C. Yao, and L.-C. Zeng, "A proximal method for pseudomonotone type variational-like inequalities," Taiwanese Journal of Mathematics, vol. 10, no. 2, pp. 497-513, 2006.

[4] L. C. Zeng, L. J. Lin, and J. C. Yao, "Auxiliary problem method for mixed variational-like inequalities," Taiwanese Journal of Mathematics, vol. 10, no. 2, pp. 515-529, 2006.

[5] J.-W. Peng and J.-C. Yao, "Ishikawa iterative algorithms for a generalized equilibrium problem and fixed point problems of a pseudo-contraction mapping," Journal of Global Optimization, vol. 46, no. 3, pp. 331-345, 2010. 
[6] L.-C. Zeng, S.-Y. Wu, and J.-C. Yao, "Generalized KKM theorem with applications to generalized minimax inequalities and generalized equilibrium problems," Taiwanese Journal of Mathematics, vol. 10, no. 6, pp. 1497-1514, 2006.

[7] J.-W. Peng and J.-C. Yao, "Some new extragradient-like methods for generalized equilibrium problems, fixed point problems and variational inequality problems," Optimization Methods and Software, vol. 25, no. 5, pp. 677-698, 2010.

[8] L.-C. Ceng, C. Lee, and J.-C. Yao, "Strong weak convergence theorems of implicit hybrid steepestdescent methods for variational inequalities," Taiwanese Journal of Mathematics, vol. 12, no. 1, pp. 227$244,2008$.

[9] J.-W. Peng and J.-C. Yao, "Strong convergence theorems of iterative scheme based on the extragradient method for mixed equilibrium problems and fixed point problems," Mathematical and Computer Modelling, vol. 49, no. 9-10, pp. 1816-1828, 2009.

[10] L.-C. Ceng, Q. H. Ansari, and J.-C. Yao, "Viscosity approximation methods for generalized equilibrium problems and fixed point problems," Journal of Global Optimization, vol. 43, no. 4, pp. 487-502, 2009.

[11] J.-W. Peng and J.-C. Yao, "Some new iterative algorithms for generalized mixed equilibrium problems with strict pseudo-contractions and monotone mappings," Taiwanese Journal of Mathematics, vol. 13, no. 5, pp. 1537-1582, 2009.

[12] B. Martinet, "Régularisation d'inéquations variationnelles par approximations successives," Revue Franç'Informatique et de Recherche Opérationnelle, vol. 4, pp. 154-158, 1970.

[13] F. Kohsaka and W. Takahashi, "Strong convergence of an iterative sequence for maximal monotone operators in a Banach space," Abstract and Applied Analysis, vol. 2004, no. 3, pp. 239-249, 2004.

[14] J. W. Peng and J. C. Yao, "A new hybrid-extragradient method for generalized mixed equilibrium problems and fixed point problems and variational inequality problems," Taiwanese Journal of Mathematics, vol. 12, pp. 1401-1433, 2008.

[15] R. T. Rockafellar, "Monotone operators and the proximal point algorithm," SIAM Journal on Control and Optimization, vol. 14, no. 5, pp. 877-898, 1976.

[16] L. C. Ceng, T. C. Lai, and J. C. Yao, "Approximate proximal algorithms for generalized variational inequalities with paramonotonicity and pseudomonotonicity," Computers $\mathcal{E}$ Mathematics with Applications, vol. 55, no. 6, pp. 1262-1269, 2008.

[17] L.-C. Ceng, S.-Y. Wu, and J.-C. Yao, "New accuracy criteria for modified approximate proximal point algorithms in Hilbert spaces," Taiwanese Journal of Mathematics, vol. 12, no. 7, pp. 1691-1705, 2008.

[18] O. Güler, "On the convergence of the proximal point algorithm for convex minimization," SIAM Journal on Control and Optimization, vol. 29, no. 2, pp. 403-419, 1991.

[19] L.-C. Ceng and J.-C. Yao, "Generalized implicit hybrid projection-proximal point algorithm for maximal monotone operators in Hilbert space," Taiwanese Journal of Mathematics, vol. 12, no. 3, pp. 753-766, 2008.

[20] L. C. Zeng and J. C. Yao, "An inexact proximal-type algorithm in Banach spaces," Journal of Optimization Theory and Applications, vol. 135, no. 1, pp. 145-161, 2007.

[21] L. C. Ceng and J. C. Yao, "Approximate proximal algorithms for generalized variational inequalities with pseudomonotone multifunctions," Journal of Computational and Applied Mathematics, vol. 213, no. 2, pp. 423-438, 2008.

[22] M. V. Solodov and B. F. Svaiter, "Forcing strong convergence of proximal point iterations in a Hilbert space," Mathematical Programming., vol. 87, no. 1, pp. 189-202, 2000.

[23] S. Kamimura and W. Takahashi, "Strong convergence of a proximal-type algorithm in a Banach space," SIAM Journal on Optimization, vol. 13, no. 3, pp. 938-945, 2003.

[24] X. Qin and Y. Su, "Strong convergence theorems for relatively nonexpansive mappings in a Banach space," Nonlinear Analysis. Theory, Methods E Applications, vol. 67, no. 6, pp. 1958-1965, 2007.

[25] L. C. Ceng, A. Petruşel, and S. Y. Wu, "On hybrid proximal-type algorithms in Banach spaces," Taiwanese Journal of Mathematics, vol. 12, no. 8, pp. 2009-2029, 2008.

[26] I. Cioranescu, Geometry of Banach Spaces, Duality Mappings and Nonlinear Problems, vol. 62 of Mathematics and Its Applications, Kluwer Academic Publishers, Dordrecht, The Netherlands, 1990.

[27] W. Takahashi, Nonlinear Functional ASnalysis, Yokohama Publishers, Yokohama, Japan, 2000, Fixed point theory and Its application.

[28] Y. I. Alber, "Metric and generalized projection operators in Banach spaces: properties and applications," in Theory and Applications of Nonlinear Operators of Accretive and Monotone Type, A. G. Kartsatos, Ed., vol. 178 of Lecture Notes in Pure and Applied Mathematics, pp. 15-50, Dekker, New York, NY, USA, 1996. 
[29] Y. I. Alber and S. Reich, "An iterative method for solving a class of nonlinear operator equations in Banach spaces," Panamerican Mathematical Journal, vol. 4, no. 2, pp. 39-54, 1994.

[30] Y. Alber and S. Guerre-Delabriere, "On the projection methods for fixed point problems," Analysis, vol. 21, no. 1, pp. 17-39, 2001.

[31] S. Reich, "A weak convergence theorem for the alternating method with Bregman distances," in Theory and Applications of Nonlinear Operators of Accretive and Monotone Type, S. Reich, Ed., vol. 178 of Lecture Notes in Pure and Applied Mathematics, pp. 313-318, Marcel Dekker, New York, NY, USA, 1996.

[32] D. Butnariu, S. Reich, and A. J. Zaslavski, "Asymptotic behavior of relatively nonexpansive operators in Banach spaces," Journal of Applied Analysis, vol. 7, no. 2, pp. 151-174, 2001

[33] D. Butnariu, S. Reich, and A. J. Zaslavski, "Weak convergence of orbits of nonlinear operators in reflexive Banach spaces," Numerical Functional Analysis and Optimization, vol. 24, no. 5-6, pp. 489-508, 2003.

[34] Y. Censor and S. Reich, "Iterations of paracontractions and firmly nonexpansive operators with applications to feasibility and optimization," Optimization, vol. 37, no. 4, pp. 323-339, 1996.

[35] S.-Y. Matsushita and W. Takahashi, "A strong convergence theorem for relatively nonexpansive mappings in a Banach space," Journal of Approximation Theory, vol. 134, no. 2, pp. 257-266, 2005.

[36] E. Blum and W. Oettli, "From optimization and variational inequalities to equilibrium problems," The Mathematics Student, vol. 63, no. 1-4, pp. 123-145, 1994.

[37] P. L. Combettes and S. A. Hirstoaga, "Equilibrium programming in Hilbert spaces," Journal of Nonlinear and Convex Analysis, vol. 6, no. 1, pp. 117-136, 2005.

[38] W. Takahashi and K. Zembayashi, "Strong and weak convergence theorems for equilibrium problems and relatively nonexpansive mappings in Banach spaces," Nonlinear Analysis. Theory, Methods $\mathcal{E}$ Applications, vol. 70, no. 1, pp. 45-57, 2009.

[39] S. S. Zhang, "Shrinking projection method for solving generalized equilibrium problem, variational inequality and common fixed point in Banach spaces with applications," to appear in Science in China A: Mathematice.

[40] R. T. Rockafellar, "On the maximality of sums of nonlinear monotone operators," Transactions of the American Mathematical Society, vol. 149, pp. 75-88, 1970. 